\title{
Maintaining Treatment Integrity in the Face of Crisis: A Treatment Selection Model for Transitioning Direct ABA Services to Telehealth
}

\author{
Kristine A. Rodriguez ${ }^{1}$
}

Published online: 18 May 2020

(C) Association for Behavior Analysis International 2020

\begin{abstract}
With health care funders' increasing approval of telehealth service as an emergency measure to provide continuity of care during the COVID-19 crisis, practicing behavior analysts have an unprecedented opportunity to demonstrate that essential, medically necessary behavior-analytic services can be provided via telehealth in a manner that maintains treatment integrity and produces meaningful client outcomes. This telehealth treatment selection guide was designed to assist practicing behavior analysts in determining an appropriate protocol for the delivery of 1:1 telehealth service (i.e., a behavior technician providing instruction directly to a client, with or without the assistance of the client's caregiver, through videoconferencing). This tool aims to help behavior analysts make thoughtful clinical decisions to maintain continuity of care for the vulnerable population with autism spectrum disorders, while adhering to safety measures that provide protection to society.
\end{abstract}

Keywords COVID-19 $\cdot$ social distancing $\cdot$ telehealth $\cdot$ treatment integrity

At the time of publication of this article, a global pandemic (COVID-19) has necessitated drastic changes to societal structures the world over: Entire states, provinces, and even countries have been placed under government orders for citizens to remain at home, allowing only essential services to continue. The Centers for Disease Control and Prevention (CDC) and the World Health Organization (WHO) have released guidance on community mitigation strategies (CDC, n.d., WHO, 2020); limiting close social contact (i.e., social distancing) has previously been identified as critical to slowing the spread of pandemics and is ultimately expected to save countless lives (CDC, 2017). In the United States, a national state of

\section{Editor's Note}

This manuscript is being published on a highly expedited basis, as part of a series of emergency publications designed to help practitioners of applied behavior analysis take immediate action to mitigate and adjust to the COVID-19 crisis. This article was submitted on March 27, 2020, and received final acceptance on March 29, 2020. The journal would like to especially thank Jennifer Zarcone for her insightful and expeditious review of this manuscript. The views and strategies suggested by the articles in this series do not represent the positions of the Association for Behavior Analysis International or Springer Nature.

Kristine A. Rodriguez

kristinerodriguez@autismlearningpartners.com

1 Department of Clinical Development and Outcomes, Autism Learning Partners, Pasadena, CA 91106, USA emergency was declared on March 13, 2020. During the state of emergency, applied behavior analysis (ABA) as a treatment for autism has generally been allowed to continue as an essential health service. However, as social-distancing restrictions tighten, the entire field is beginning to vet the feasibility and appropriateness of transitioning-at least temporarily- to telehealth, a service delivery model that for the purpose of this article will focus on live (synchronous) videoconferencing to deliver behavior-analytic services (i.e., the behavior therapist delivers instruction to the client via videoconferencing).

There exists a growing body of research dedicated to evaluating the effectiveness of telehealth as a viable modality for delivering behavior-analytic services, encompassing a variety of interventions from teaching functional communication skills (Simacek, Dimian, \& McComas, 2017) to treating problem behavior (Lindgren et al., 2016). Researchers have written about the need to consider ethics when implementing services via telehealth (Pollard, Karimi, \& Ficcaglia, 2017; Romani \& Schieltz, 2017). Pollard et al. (2017) published a thorough and practical article outlining clinical and business infrastructure considerations in the development of ethical telehealth services (Pollard et al., 2017). Additionally, systematic reviews of telehealth as a model for delivery of behavior-analytic services have been conducted (Ferguson, Craig, \& Dounavi, 2019; Tomlinson, Gore, \& McGill, 2018). A distinction between the services outlined in the majority of these articles and the model 
presented here is the focus on direct service delivery. As much of the literature focuses on parent consultation between a Board Certified Behavior Analyst (BCBA) and the caregiver, there is limited guidance for a direct model (i.e., beyond parent consult, working directly with the client from a remote location). There also is no published guidance the author is aware of to inform a systematic process for vetting the appropriateness of different telehealth models or structures based on clients' and caregivers' presentation of various skill sets and individual needs. As health care funders quickly move to allow telehealth service as an emergency provision to facilitate continuity of care during this crisis, practitioners have an unprecedented opportunity to demonstrate that essential, medically necessary behavior-analytic services can be provided remotely in a manner that maintains treatment integrity and upholds meaningful client outcomes.

This telehealth treatment selection guide was designed to assist BCBAs in determining an appropriate protocol for delivery of direct, 1:1 telehealth services - specifically, that which involve a behavior technician (BT) providing instruction directly to a client, with or without the assistance of the client's caregiver, using videoconferencing technology. Admittedly, this modality may not be a clinical fit for all clients, and some programs may be more readily modified to suit the distance model than others. This model was created in an attempt to provide a tool for BCBAs and provider organizations to take a systematic approach in determining (a) which clients are ready for immediate treatment, with minimal program modifications; (b) which clients' programs would require substantial modifications to goals, teaching procedures, or behavior intervention plans; and (c) which clients present with barriers requiring advanced problem solving to access the benefits of direct telehealth services.

This tool, like all decision models and clinical tools, is not intended as a replacement for sufficient clinical judgment and training on the part of the BCBA. That is, decision models cannot give directives for what to do; they merely provide a framework to aid in taking a structured approach during the decision-making process. All treatment decisions must then be evaluated carefully with data and adjusted appropriately, as is always the case in ABA services, regardless of whether telehealth is being used.

The treatment guide consists of two components: a brief assessment (to be conducted via telehealth consultation with a BCBA), followed by a corresponding treatment selection matrix. Both are designed to assist the BCBA in efficiently determining a potentially appropriate telehealth model, considering both the repertoires presented by the client and the caregiver, and to provide recommendations for subsequent training of the BCBA and BT.

Program Modifications Assessment for Direct (1:1) Telehealth Services (PMA) Designed to be conducted via telehealth consultation by the BCBA, the PMA entails two components: (a) an assessment of the client's ability to attend and respond to skill acquisition programs and (b) an assessment of caregivers' ability to facilitate the skill acquisition program and the behavior intervention plan(s). The components may be interspersed in any way that facilitates the BCBA's ability to complete the assessment. As with any assessment, the consulting BCBA should first obtain consent, briefly describe the purpose of the assessment, and outline the entailed procedures (Behavior Analyst Certification Board, 2014).

\section{PMA Part 1: Skill Acquisition}

Figure 1 depicts the data sheet for Part 1, skill acquisition. The BCBA first instructs the caregiver to gather any required materials and reinforcers and then requests the caregiver to direct the client to attend to the screen (i.e., the synchronous video display of the BCBA). The BCBA attempts to run several client goals: three that have been previously mastered and generalized across people and/or settings, three that have met mastery criteria under strict stimulus conditions but are not yet meaningfully generalized, and three that are in acquisition or in progress. While running these programs, the BCBA records clinical impressions across the following fields: the number of redirections needed for the client to attend to the screen/BCBA, the number of minutes and trials to which the client attends, the least intrusive successful prompts, and the schedule of reinforcement required to maintain correct responding. The BCBA should additionally note the caregiver's ability to prompt, redirect, and deliver reinforcement.

\section{PMA Part 2: Behavior Management}

Figure 2 depicts the data sheet for Part 2, behavior management. The BCBA starts by reviewing the intervention plan with the caregiver. During the review, the BCBA guides the caregiver to create a naturalistic routine, during which time the caregiver implements antecedent strategies and prompts replacement behaviors. Next, the BCBA guides the caregiver to create the contingencies to evoke moderate-intensity (i.e., manageable) problem behavior. Care should be taken to avoid triggering severe problem behavior that cannot be safely managed by the caregiver. The BCBA provides real-time feedback and guidance to the caregiver as they work to implement the behavior plan. The BCBA assesses whether the caregiver can implement all strategies within the plan independently, with repeated reminders, or whether the caregiver is unable to implement the plan, even with prompting. 


\section{Part 1: SKILL ACQUISITION}

Instructions: BCBA probes up to 9 goals (A: 3 mastered/generalized; B: 3 technically meeting mastery criteria in session, but not generalized; C: 3 in-progress goals). If fewer than 3 available in any category, run those that are available.

Ask caregiver to provide as many redirections as needed for client to attend to screen/directions. Direct caregiver on prompting and reinforcement strategies/schedules. (BCBA provides instruction/discriminative stimulus (SD); caregiver provides prompt/reinforcement.)

\begin{tabular}{|c|c|c|c|c|c|c|c|c|c|}
\hline & \multirow[b]{2}{*}{\begin{tabular}{|l} 
Target \\
(brief)
\end{tabular}} & \multirow[b]{2}{*}{\begin{tabular}{|l|} 
Attending \\
Redirections \\
needed to \\
attend (\#)
\end{tabular}} & \multirow[b]{2}{*}{\begin{tabular}{|l|} 
Attending \\
Trials or \\
minutes \\
attended \\
without \\
prompting \\
(\#) \\
\end{tabular}} & \multirow[b]{2}{*}{\begin{tabular}{|l|} 
Prompting \\
Least intrusive \\
successful prompt \\
type
\end{tabular}} & \multirow[b]{2}{*}{\begin{tabular}{|l|} 
Reinforcement \\
SR) \\
Schedule of SR+ \\
required (e.g., 1:1, \\
FRS, etc.)
\end{tabular}} & \multicolumn{4}{|c|}{ Mark $x$ in one column for each goal } \\
\hline & & & & & & \begin{tabular}{|l} 
Minimal \\
modifications \\
needed
\end{tabular} & $\begin{array}{l}\text { Requires modifications } \\
\text { to skill acquisition } \\
\text { program/behavior } \\
\text { intervention plan (BIP) } \\
\text { (see recommendations) }\end{array}$ & \begin{tabular}{|l} 
Requires caregiver \\
coaching (see \\
recommendations)
\end{tabular} & \begin{tabular}{|l|} 
Requires advanced \\
modifications \\
(see recommendations)
\end{tabular} \\
\hline \multicolumn{10}{|l|}{ A Goal 1 } \\
\hline \multicolumn{10}{|l|}{ A Goal 2} \\
\hline \multicolumn{10}{|l|}{ A Goal 3} \\
\hline \multicolumn{10}{|l|}{ B Goal 1} \\
\hline \multicolumn{10}{|l|}{ B Goal 2} \\
\hline \multirow{2}{*}{\multicolumn{10}{|c|}{ B Goal 3}} \\
\hline & & & & & & & & & \\
\hline \multicolumn{10}{|l|}{ C Goal 1} \\
\hline \multicolumn{10}{|l|}{ C Goal 2} \\
\hline C Goal 3 & & & & & & & & & \\
\hline
\end{tabular}

Fig. 1 Program modifications assessment for direct (1:1) telehealth services (PMA), Part 1: skill acquisition

\section{Telehealth Model Selection Matrix}

The BCBA uses the telehealth model selection matrix (Figure 3) to help guide him or her in assessing the client's and caregiver's readiness for telehealth services by considering the extent to which program modifications and caregiver coaching are needed to support client progress. Based on the results from the PMA, along with information about the client's and caregiver's repertoires, the BCBA uses the matrix to assess what he or she predicts will be the most appropriate telehealth treatment model. The matrix serves as a tool to categorize clients' repertoires based on their prompting needs, responses to reinforcement schedules, and intensity of problem behaviors. The tool further helps the BCBA assess caregivers' repertoires based on their ability to facilitate session structure and to manage problem behaviors. The selection of repertoires leads to the selection of a corresponding treatment structure. Finally, the tool lists potential training topics for BCBAs and BTs in support of the selected model. Figure 4 provides an example of a template that may be used to summarize modifications to the skill acquisition program, modifications to the behavior intervention plan, or additional goals for caregivers.

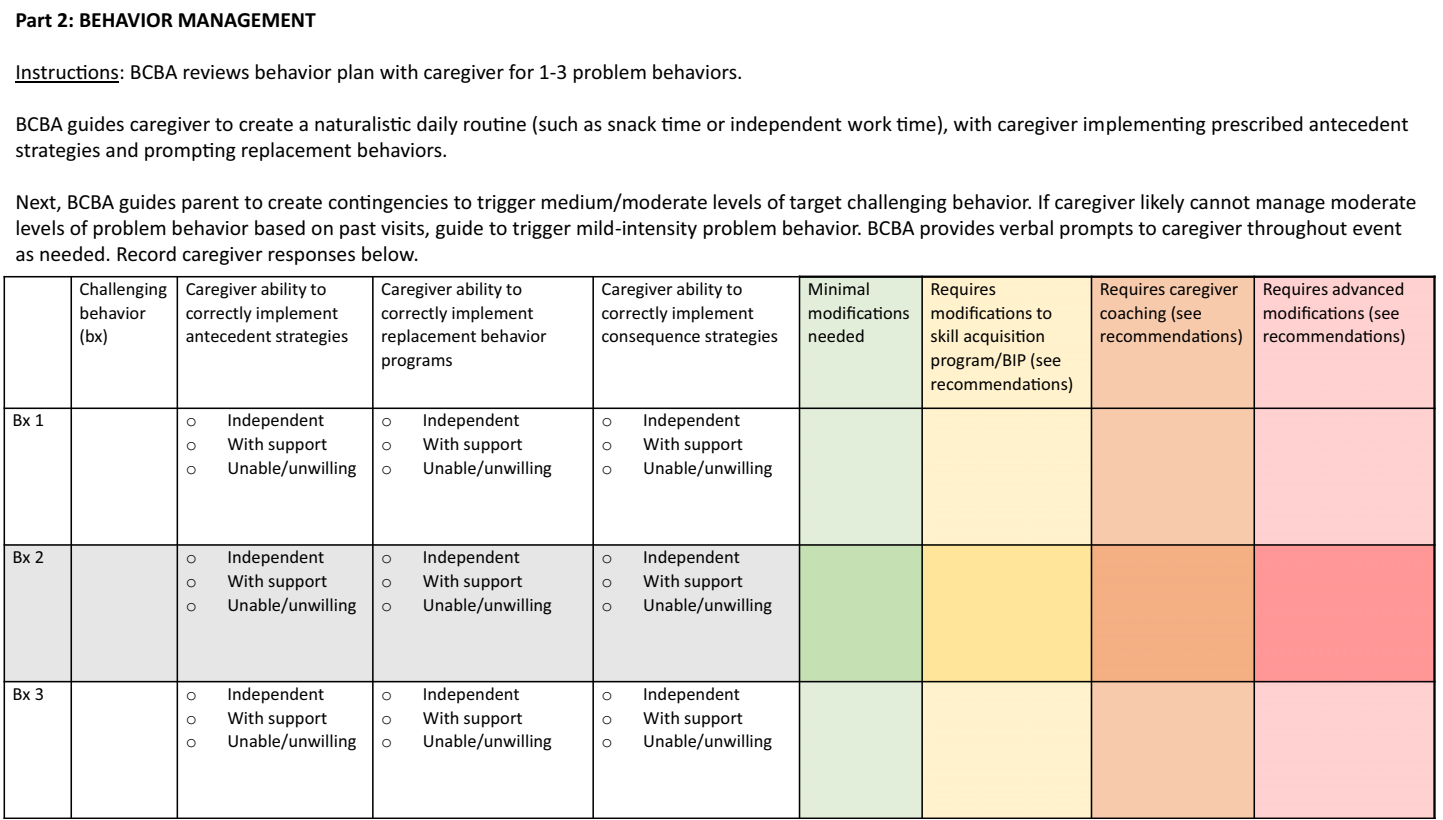

Fig. 2 Program modifications assessment for direct (1:1) telehealth services (PMA), Part 2: behavior management 
Telehealth Model Selection Matrix

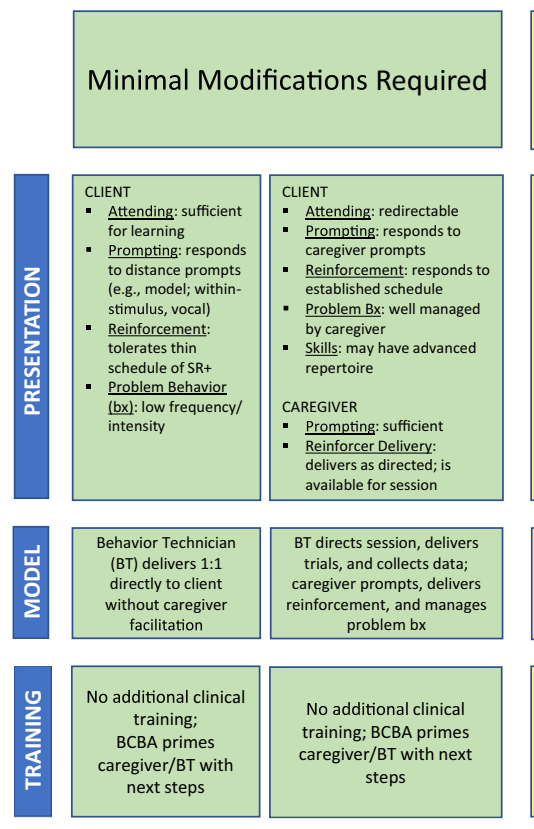

Modifications to Skill Acquisition/Behavior Intervention Plan (BIP) Required
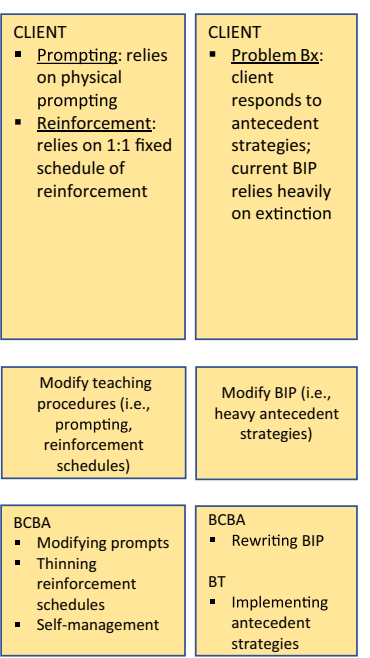

Caregiver Coaching Required
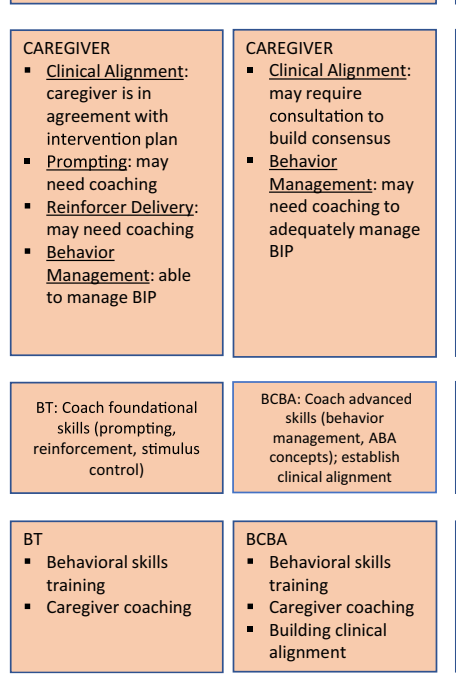

BCBA training Building clinical alignment
- Behavioral skills

- Caregiver coachin
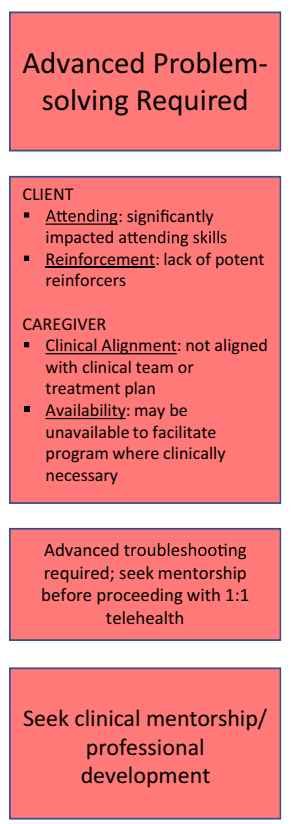

Fig. 3 Telehealth model selection matrix

\section{Minimal Modifications}

Presentation Clients who are well suited for minimal program modifications in a telehealth modality likely demonstrate moderate to strong attending skills. The number of minutes or trials attended in the assessment should indicate that the client can engage in learning opportunities successfully. The exact duration of attending will vary based on the age of the learner, individual learner characteristics, and the needs of the program. The client demonstrates the ability to respond to distance prompts - that is, prompts that would be delivered by the BT via video meeting. It is optimal that the client does not rely on physical prompting to respond to learning trials. Alternatively, the client may require physical prompts, but in that case, the BCBA notes during the assessment that the caregiver is adept at delivering these consistently and is ideally able to fade prompts with coaching from the BT. The client demonstrates the ability to self-manage a reinforcement system or to tolerate delayed reinforcement, or the client responds consistently to a reinforcement schedule delivered by the caregiver. In some instances, the repertoire presented by the learner may include an advanced skill set (e.g., a client being treated primarily for impacted social skills). Problem behavior may be infrequent or low intensity, or alternatively, it may be moderate but well managed by the caregiver.

Indicated Model Based on the presenting repertoire described previously, the client may be considered a strong candidate for telehealth services requiring few or minimal modifications. If the client attends well, responds to distance prompts, tolerates delayed reinforcement, and engages in only mild or infrequent problem behavior, he or she may be a candidate for remote direct instruction (i.e., client and BT) without requiring facilitation from the caregiver. If the client demonstrates a more impacted repertoire (e.g., difficulty attending, requiring more intrusive prompting, exhibiting challenging behaviors), but the caregiver demonstrates a strong repertoire to redirect, prompt, provide reinforcement, and manage challenging behavior, then the client may also be a strong candidate for a telehealth program with minimal modifications. In this case, the caregiver acts as the facilitator in person, while the BT provides the direct service by delivering instructions; coaching the caregiver to prompt, deliver reinforcement, and navigate the behavior plan; and collecting data.

Indicated Training Given that minimal modifications are required, it follows that minimal training specific to this model is required. Basic procedural support for setting up video sessions, maintaining confidentiality, and assessing treatment integrity should be provided. Continued program monitoring is required to assess the integrity of this expanded modality of services and provide any needed support. Training on general safety, confidentiality, and technology setup are prerequisite skills or needed training topics, but they are outside the scope of this tool. For guidance on developing and troubleshooting telehealth services, the reader may consult guidelines such as those submitted by Lee et al. (2015). 


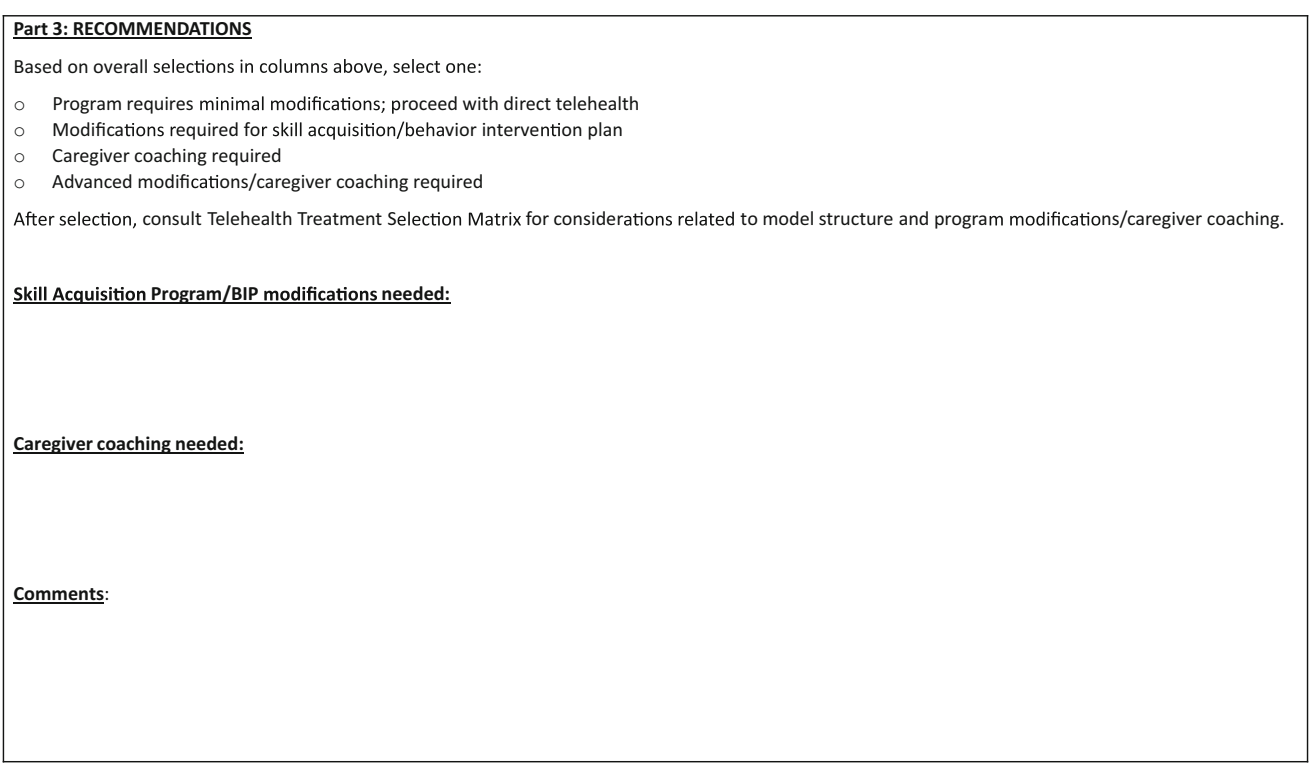

Fig. 4 Example template of summary of recommendations

\section{Modifications to Skill Acquisition/Behavior Intervention Plan}

Presentation More substantial modifications are likely needed for skill acquisition programs when the client demonstrates a heavy reliance on physical prompting and continuous schedules of reinforcement in order to respond correctly to learning trials. This increases the reliance on skillful, consistent facilitation by the caregiver. Additionally, if the behavior intervention plan relies heavily on consequent strategies or extinction, there is a greater reliance on the caregiver's ability to provide consistently accurate implementation of those strategies. Given that some caregivers report dissatisfaction or discomfort with some extinction procedures, modification to the behavior intervention plan (if appropriate) may lead to improved social validity and improved treatment adherence by the caregiver, as well as an overall reduced intensity of challenging behavior throughout telehealth sessions.

Model For the client who demonstrates reliance on intrusive prompts and dense schedules of reinforcement, the BCBA is advised to consider adjusting the focus of the skill acquisition program to promote attending to distance prompts, and to program tolerance to thinner, more naturalistic schedules of reinforcement. The addition of a self-management program may also result in a significant benefit from telehealth services. For example, teaching the client to travel to the caregiver to recruit reinforcement once earned may improve the client's likelihood of obtaining reinforcement under generalized contingencies. Such programming could also provide a benefit to the caregiver, who may need to attend to other priorities that conflict with fully facilitating sessions. Additionally, it may be appropriate to temporarily place certain program goals on hold, to instead focus on the client's goals that are more readily addressed with a telehealth modality.

For caregivers who demonstrate a reluctance to implement extinction or other consequence-based strategies consistently, the matrix suggests that modification to the behavior plan may be appropriate. Targeted, function-based antecedent strategies may have higher social validity and be more acceptable to some caregivers. For example, a BCBA may elect to omit extinction from the behavior intervention plan and instead focus heavily on caregiver implementation of noncontingent reinforcement throughout telehealth sessions.

Training Providing behavior-analytic services in person allows the BCBA and BT to benefit from strong stimulus control that may not be present in the home setting. Some BCBAs may not be well versed in least intrusive prompting methods for effective procedures for thinning schedules of reinforcement. To address these needs, a BCBA may benefit from learning strategies to facilitate transfer of stimulus control from the in-person instructional setting to the telehealth setting. Training on how to successfully implement a variety of effective, less intrusive prompting strategies, as well as training in reinforcement schedules and thinning and selfmanagement protocols, may be beneficial.

Due to the general effectiveness of consistently used extinction procedures, a BCBA may rely heavily on extinction procedures when the team includes BTs who are skilled in implementing extinction. In cases of heavy reliance on extinction procedures, the BCBA may be less fluent in the use of certain effective antecedent strategies. Additional training focusing on function-specific antecedent strategies and programs targeting replacement behaviors may be needed. 


\section{Caregiver Coaching}

Presentation There are two particularly relevant aspects of caregiver repertoires: (a) the implementation skill set and (b) alignment with the clinical direction of the program. In the event that the caregiver and clinical team are in agreement on the focus and direction of the program and the caregiver is relatively well trained, the caregiver may simply require coaching to sharpen his or her skill set for foundational skills related to facilitating the treatment plan (e.g., redirection, prompting, and delivery of reinforcement). In other cases, the caregiver and clinical team may be well aligned, but the caregiver requires more advanced training and coaching (e.g., implementation of behavior management strategies or specific prompting and fading strategies). Alternatively, if there is a misalignment between the caregiver and the clinical team, there may be the need to provide additional consultation from the BCBA or clinical director in order to strengthen the alignment with the clinical direction of the treatment plan or make reasonable adjustments to the plan where appropriate.

Model In some cases, if the BT has demonstrated the ability to successfully coach thespecifictargeted skills, heorshemaybe abletodelivercaregivercoachingforsharpeningbasiccaregiverskills.Ifmorecomplexskillsmustbetaught,especiallygiven the challengepresented by aremotebehavioral-skills-training model, consultation with the BCBA directly is most appropriate. Ininstances whereadditional consultation isneeded tocreate strongerclinical team-caregiver alignmentor make appropriateadjustmentstotheprogramming, consultationshouldbe provided by the BCBA or clinical director. In some instances, multipleconsultationsessionsmaybeneededbeforeitisadvisable to move forward with direct telehealth services between theBTand the caregiver/client.

Training In either model, the BCBA should ensure that he or she and the BT have received training in effective consultation, particularly in the use of a behavioral-skills-training model. Additionally, the BCBA should seek training in working with caregivers to create a treatment plan that supports the client's self-determination and is strongly aligned with the family's values and priorities, as this has been shown to improve caregiver adherence to treatment and overall success with the intervention (Gould, Tarbox, \& Coyne, 2018; Martin, Williams, Haskard, \& Dimatteo, 2005).

\section{Advanced Problem Solving}

Presentation Clients whose cases comprise the final category may present with particularly challenging repertoires for the telehealth model. These presentations may include extremely limited joint attention, the necessity of frequent redirection, a scarcity of potent reinforcers, and the presence of intense or dangerous problem behaviors. Additionally, caregivers may be unavailable or unable to facilitate services or may be opposed to the treatment plan.

Model Some clients may present with unique challenges or barriers to a quick rollout of the telehealth model for 1:1 services. It is beyond the scope of this matrix to recommend, concisely, the path forward for this group of clients. It is critically important, however, that care is taken to explore potential solutions and innovations in service delivery to overcome the barriers presented by this group. In no way should this tool be used to suggest that these clients may not benefit from telehealth services outright. On the contrary, these clients may be those most in need of continuity of care and maintained service delivery during these times of unprecedented disruption to their daily routines and social structures. It is, however, likely that the need for more robust consultation with a BCBA or more tailored troubleshooting for teaching these clients remotely will lead to a longer period of preparation before beginning 1:1 telehealth services, compared to the models discussed up to this point that allow for more immediate implementation of a telehealth model.

Training The professional development and training required to serve this group of clients will vary widely based on the individual barriers to treatment and relevant risks. These include, but are by no means limited to, advanced training in creating caregiver alignment, training in technological solutions for engaging clients remotely, and maintaining safety. BCBAs should seek mentorship from clinicians experienced in managing the particular barriers presented by the client's (or caregiver's) repertoire, as well as from BCBAs with substantial experience treating challenging cases over telehealth.

\section{Discussion}

\section{Potential Limitations}

This clinical tool was developed under extremely timesensitive circumstances and, like all tools, is not without limitations. First, although the content of this tool consists of evidence-based practices, this tool, itself, has not yet undergone empirical field testing. Therefore, the potential added benefit of using this tool has not been confirmed by research. It would be valuable to conduct formal research on the effectiveness of this model as a practical tool for transitioning clients historically served in person to a fully telehealth model.

A second potential limitation relates to the very use of telehealth for the delivery of direct ABA services. Multiple studies, such as those summarized by Healy and Lydon (2013), support that a robust intensity of direct service (typically falling between 20 and 40 hours per week) is associated with optimal outcomes in behavior-analytic treatment. Although caregiver 
consultation is a valuable element to incorporate into behavioranalytic programs, the literature does not suggest that a caregiverled model would outperform a model implemented directly by a professional trained extensively in ABA. A potential limitation of telehealth services in general, particularly in models indicating caregiver facilitation, is that the intensity of services may be particularly limited based on caregiver availability. This is problematic, in that the outcome literature does not suggest that some small amount of treatment is better than nothing. Therefore, care should be taken to maintain an intensity of services that reflects best practice literature and is matched to the individual needs of the client, even while pursuing a telehealth model. This is why it is so critical to move clients toward a model that lessens reliance on caregiver facilitation (i.e., through less intrusive prompting and thinned schedules of reinforcement). Although this potential limitation is a grave concern, if in-person services are not possible due to the COVID-19 pandemic, it seems plausible that delivery of telehealth services is likely to produce some benefits to the client over no services at all.

A third limitation is that this tool is not (and is not intended to be) a comprehensive tool encompassing all aspects of clinical and business infrastructure related to rolling out telehealth services. It is simply one tool, developed and disseminated rapidly, for the purposes of helping fellow BCBAs take a structured approach to decision making during a crisis. Many others are also developing useful resources, including the Behavior Analyst Certification Board (2020), the Behavioral Health Center of Excellence (Blanco, Meisels, Blair, \& Leonard, 2020), and the Council of Autism Service Providers (n.d.), among others. These will continue to be highly valuable resources for the field even after the current crisis subsides.

Finally, a critical limitation of any telehealth model is the potential to limit care for those most in need. This not only pertains to those who present with the most intense problem behaviors and the poorest attending skills but also to those whose socioeconomic status or physical geographical location limits access to a reliable Internet connection or to Internetenabled devices to facilitate a secure video connection. These same factors may limit a caregiver's availability to facilitate as well, especially amid a crisis that has led to some caregivers managing multiple contingencies - as caregiver, employee, homeschooler, and more. However, it may be the case that implementing telehealth services in appropriate instances will allow for the allocation of resources to the smaller subset of clients for whom in-person service delivery is essential to maintain their safety (e.g., reducing the number of clients being seen in a clinic, or reducing the number of clients and homes with which a BT comes in contact).

\section{Critical Opportunity}

Even in the face of the limitations discussed previously, the current reality provides a unique opportunity for BCBAs to fulfill a very immediate need - to safely provide continuity of essential care for clients while complying with regional socialdistancing mandates - while potentially fulfilling a broader and longer lasting purpose. When all social functioning has returned to normal — or the new picture of normal - there will still be clients to serve who do not have access to consistent, in-person ABA services. A number of logistical challenges can be resolved by delivering telehealth services. If through this crisis, the field of ABA can uncover tools to responsibly transition to telehealth services in a way that maintains clinical integrity and desired client outcomes, there are a number of potential benefits. Immediately, clients will continue to access services, and countless jobs will be saved during the greatest crisis of a generation. In the long run, ultimately the field may emerge with a powerful new skill set to conquer the clinical and logistical challenges of the future.

Acknowledgements The author wishes to thank Allyson Kroneberger, Jo Anna Mazzacane, Courtney Nolan, Sarah Torgrimson, Dan Matas, and Dr. Gina Chang for their collaboration and encouragement.

\section{Compliance with Ethical Standards}

Conflict of Interest The author declares she has no conflicts of interest.

Ethical Approval This article was written in compliance with international regulations regarding research; this article contains no studies involving human participants or animal subjects.

\section{References}

Behavior Analyst Certification Board. (2014). Professional and ethical compliance code for behavior analysts. Retrieved from http://www. bacb.com/wp-content/uploads/BACB-Compliance-Code-english_ 190318.pdf

Behavior Analyst Certification Board. (2020). Ethics guidance for ABA providers during Covid-19 pandemic. Retrieved from https://www. bacb.com/ethics-guidance-for-aba-providers-during-covid-19pandemic-2/

Blanco, S., Meisels, M., Blair, B. J., \& Leonard, L. (2020). Telehealth: Evidence-based practice in the time of COVID-19 [Web log post]. Retrieved from https://bhcoe.org/category/blog/applied-behavioranalysis/

Centers for Disease Control and Prevention. (n.d.). Implementation of mitigation strategies for communities with local Covid-19 transmission. Retrieved from https://www.cdc.gov/coronavirus/ 2019-ncov/downloads/community-mitigation-strategy.pdf

Centers for Disease Control and Prevention. (2017). Community mitigation guidelines to prevent pandemic influenza. Morbidity and Mortality Weekly Report, 66(1). Retrieved from https://stacks.cdc. gov/view/cdc/45220

The Council of Autism Service Providers. (n.d.). Telehealth and crisis management guidelines. Retrieved from https://casproviders.org/ telehealth-and-crisis-management-guidelines/

Ferguson, J., Craig, E. A., \& Dounavi, K. (2019). Telehealth as a model for providing behaviour analytic interventions to individuals with autism spectrum disorder: A systematic review. Journal of Autism 
and Developmental Disorders, 49(2), 582-616. https://doi.org/10. 1007/s10803-018-3724-5.

Gould, E. R., Tarbox, J., \& Coyne, L. (2018). Evaluating the effects of acceptance and commitment training on the overt behavior of parents of children with autism. Journal of Contextual Behavioral Science, 7, 81-88. https://doi.org/10.1016/j.jcbs.2017.06.003.

Healy, O., \& Sinéad, L. (2013). Early intensive behavioural intervention in autism spectrum disorders. In M. Fitzgerald (Ed.), Recent advances in autism spectrum disorders (pp. 567-597). London, UK: IntechOpen. https://doi.org/10.5772/54274.

Lee, J. F., Schieltz, K. M., Suess, A. N., Wacker, D. P., Romani, P. W., Lindgren, S. D., et al. (2015). Guidelines for developing telehealth services and troubleshooting problems with telehealth technology when coaching parents to conduct functional analyses and functional communication training in their homes. Behavior Analysis in Practice, 8(2), 190-200. https://doi.org/10.1007/s40617-014-00312.

Lindgren, S., Wacker, D., Suess, A., Schieltz, K., Pelzel, K., Kopelman, T., et al. (2016). Telehealth and autism: Treating challenging behavior at lower cost. Pediatrics, 137(Suppl. 2), S167-S175. https://doi. org/10.1542/peds.2015-2851O.

Martin, L. R., Williams, S. L., Haskard, K. B., \& Dimatteo, M. R. (2005). The challenge of patient adherence. Therapeutics and Clinical Risk Management, 1, 189-199.

Pollard, J. S., Karimi, K. A., \& Ficcaglia, M. B. (2017). Ethical considerations in the design and implementation of a telehealth service delivery model. Behavior Analysis: Research and Practice, 17(4), 298-311. https://doi.org/10.1037/bar0000053.

Romani, P. W., \& Schieltz, K. M. (2017). Ethical considerations when delivering behavior analytic services for problem behavior via telehealth. Behavior Analysis: Research and Practice, 17(4), 312 324. https://doi.org/10.1037/bar0000074.

Simacek, J., Dimian, A. F., \& McComas, J. J. (2017). Communication intervention for young children with severe neurodevelopmental disabilities via telehealth. Journal of Autism and Developmental Disorders, 47, 744-767. https://doi.org/10.1007/s10803-016-3006-z.

Tomlinson, S. R., Gore, N., \& McGill, P. (2018). Training individuals to implement applied behavior analytic procedures via telehealth: A systematic review of the literature. Journal of Behavioral Education, 27(2), 172-222. https://doi.org/10.1007/s10864-0189292-0.

World Health Organization. (2020). Critical preparedness, readiness and response actions for COVID-19. Retrieved from https://www.who. int/emergencies/diseases/novel-coronavirus-2019/technicalguidance/critical-preparedness-readiness-and-response-actions-forcovid-19

Publisher's Note Springer Nature remains neutral with regard to jurisdictional claims in published maps and institutional affiliations. 